\title{
THE FIRST COEFFICIENT OF THE CONWAY POLYNOMIAL
}

\author{
JIM HOSTE
}

\begin{abstract}
A formula is given for the first coefficient of the Conway polynomial of a link in terms of its linking numbers. A graphical interpretation of this formula is also given.
\end{abstract}

Introduction. Suppose that $L$ is an oriented link of $n$ components in $S^{3}$. Associated to $L$ is its Conway polynomial $\nabla_{L}(z)$, which must be of the form

$$
\nabla_{L}(z)=z^{n-1}\left[a_{0}+a_{1} z^{2}+\cdots+a_{m} z^{2 m}\right] .
$$

Let $\tilde{\nabla}_{L}(z)=\nabla_{L}(z) / z^{n-1}$. In this paper we shall give a formula for $a_{0}=\tilde{\nabla}_{L}(0)$ which depends only on the linking numbers of $L$. We will also give a graphical interpretation of this formula.

It should be noted that the formula we give was previously shown to be true up to absolute value in [3]. The author wishes to thank Hitoshi Murakami for bringing Professor Hosakawa's paper to his attention.

We shall assume a basic familiarity with the Conway polynomial and its properties. The reader is referred to $[\mathbf{1}, \mathbf{2}, \mathbf{4}, 5$ and 6] for a more detailed exposition. The fact that $\nabla_{L}(z)$ has the form described above can be found in [4 or 6], for example.

1. A formula for $\tilde{\nabla}_{L}(0)$. Suppose $L=\left\{K_{1}, K_{2}, \ldots, K_{n}\right\}$ is an oriented link in $S^{3}$. Let $l_{i j}=\operatorname{lk}\left(K_{i}, K_{j}\right)$ if $i \neq j$ and define $l_{i i}=-\sum_{j=1, j \neq i}^{n} l_{i j}$. Define the linking matrix $\mathscr{L}$, or $\mathscr{L}(L)$, as $\mathscr{L}=\left(l_{i j}\right)$. Now $\mathscr{L}$ is a symmetric matrix with each row adding to zero. Under these conditions it follows that every cofactor $\mathscr{L}_{i j}$ of $\mathscr{L}$ is the same. (Recall that $\mathscr{L}_{i j}=(-1)^{i+j} \operatorname{det} M_{i j}$, where $M_{i j}$ is the $(i, j)$ minor of $\mathscr{L}$.)

THEOREM 1. Let $L$ be an oriented link of $n$ components in $S^{3}$. Then $\tilde{\nabla}_{L}(0)=\mathscr{L}_{i j}$, where $\mathscr{L}_{i j}$ is any cofactor of the linking matrix $\mathscr{L}$.

Proof. Let $F$ be a Seifert surface for $L$. We may picture $F$ as shown in Figure 1.1. Let $\left\{a_{i}\right\}$ be the set of generators for $H_{1}(F)$ shown in the figure and define the Siefert matrix $V=\left(v_{i j}\right)$ in the usual way. Namely, $v_{i j}=\operatorname{lk}\left(a_{i}^{+}, a_{j}\right)$, where $a_{i}^{+}$is obtained by lifting $a_{i}$ slightly off of $F$ in the positive direction. Then if $a_{i} \cap a_{j}=\varnothing$ we have $v_{i j}=v_{j i}=\operatorname{lk}\left(a_{i}, a_{j}\right)$. If $a_{i} \cap a_{j} \neq \varnothing$, then $\{i, j\}=\{2 k-1,2 k\}$ for some

Received by the editors August 14, 1984 and, in revised form, December 28, 1984.

1980 Mathematics Subject Classification. Primary 57M25.

Key words and phrases. Knot, link, Conway polynomial. 
$1 \leqslant k \leqslant h$ and $v_{2 k-1,2 k}=v_{2 k, 2 k-1}-1$. Hence $V$ is of the form

$$
V=\left(\begin{array}{cc}
A & B \\
B^{T} & C
\end{array}\right),
$$

where $A$ is a $2 h \times 2 h$ matrix and $C$ is a symmetric $(n-1) \times(n-1)$ matrix.

Now $a_{2 h+i-1}$ is parallel to $K_{i}$ for $i>1$. Hence $v_{2 h+i-1,2 h+j-1}=l_{i j}$ for $i \neq j$ and $i, j>1$. Furthermore,

$$
\begin{aligned}
l_{1 i} & =-\sum_{j=2}^{n} v_{2 h+i-1,2 h+j-1} \\
& =-\left(l_{i 2}+l_{i 3}+\cdots+l_{i, i-1}+v_{2 h+i-1,2 h+i-1}+l_{i, i+1}+\cdots+l_{i, n}\right) .
\end{aligned}
$$

Therefore, we have

$$
v_{2 h+i-1,2 h+i-1}=-\sum_{j=1, j \neq i}^{n} l_{i j}=l_{i, i} .
$$

Hence we have that $C$ is the $(1,1)$ minor of $\mathscr{L}$.

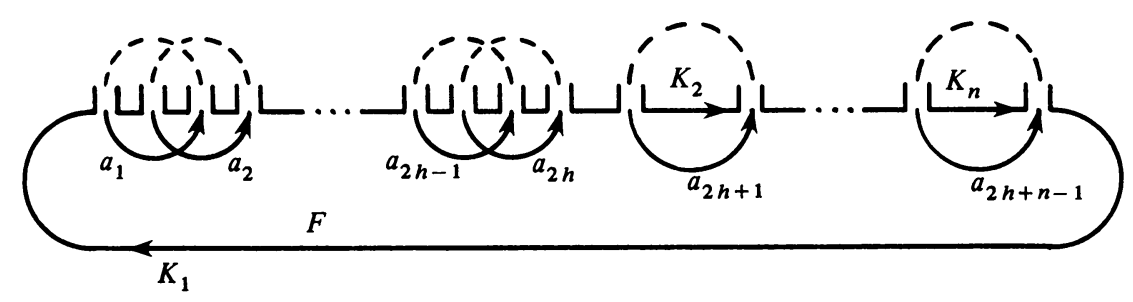

FIGURE 1.1

Now the Conway polynomial can be defined as $\nabla_{L}(z)=\operatorname{det}\left(t V-t^{-1} V^{T}\right)$, where the right-hand side of this equation is a polynomial in $z=t-t^{-1}$. Hence we have

$$
\begin{aligned}
\tilde{\nabla}_{L}\left(t-t^{-1}\right) & =\operatorname{det}\left(t V-t^{-1} V^{T}\right) /\left(t-t^{-1}\right)^{n-1} \\
& =\operatorname{det}\left(\begin{array}{cc}
t A-t^{-1} A^{T} & \left(t-t^{-1}\right) B \\
\left(t-t^{-1}\right) B^{T} & \left(t-t^{-1}\right) C
\end{array}\right) /\left(t-t^{-1}\right)^{n-1} \\
& =\operatorname{det}\left(\begin{array}{cc}
t A-t^{-1} A^{T} & \left(t-t^{-1}\right) B \\
B^{T} & C
\end{array}\right) .
\end{aligned}
$$

So,

$$
\tilde{\nabla}_{L}(0)=\operatorname{det}\left(\begin{array}{cc}
A-A^{T} & 0 \\
B^{T} & C
\end{array}\right) .
$$

But

$$
A-A^{T}=\left[\begin{array}{rrrrrrr}
0 & -1 & & & & & \\
1 & 0 & & & & 0 & \\
& & 0 & -1 & & & \\
& & 1 & 0 & & & \\
& & & & \ddots & & \\
& & 0 & & & 0 & -1 \\
& & & & & 1 & 0
\end{array}\right]
$$


so that $\tilde{\nabla}_{L}(0)=\operatorname{det} C=\mathscr{L}_{1,1}$. Since all the cofactors of $\mathscr{L}$ are equal, the theorem follows.

2. A graphical interpretation of $\tilde{\nabla}_{L}(0)$. Let $\Gamma(L)$ be the complete graph with $n$ vertices. Label the vertices $K_{1}, \ldots, K_{n}$ and label the edge connecting $K_{i}$ and $K_{j}$ with their linking number $l_{i j}$. Let $G$ be the set of all subgraphs of $\Gamma$ consisting of $n-1$ distinct edges together with their vertices. Let $T$ be the subset of $G$ consisting of those graphs which are trees. If $g \in G$ let $\bar{g}$ be the product of the $n-1$ linking numbers associated to the edges of $g$.

THEOREM 2. Suppose $L$ is an oriented link in $S^{3}$ with $n$ components. Then $\tilde{\nabla}_{L}(0)=(-1)^{n-1} \sum_{g \in T} \bar{g}$.

Proof. It follows from Theorem 1 that $\tilde{\nabla}_{L}(0)$ is a finite sum of terms, where each term is a product of $n-1$ linking numbers together with some integer coefficient. Now each term is actually the product of $n-1$ distinct linking numbers. For consider some $l_{i j}$. It appears in only four entries of $\mathscr{L}$, namely $l_{i i}, l_{i j}, l_{j i}$, and $l_{j j}$. Hence $l_{i j}$ appears only once in the $(i, i)$ minor of $\mathscr{L}$ and so cannot appear to any power greater than one in $\mathscr{L}_{i i}$. Thus we have shown that

$$
\tilde{\nabla}_{L}(0)=\mathscr{L}_{i j}=(-1)^{n-1} \sum_{g \in G} \varepsilon(g) \bar{g}
$$

where $\varepsilon(g)$ is some integer.

We want to show that $\varepsilon(g)$ is one if $g$ is a tree and zero otherwise.

Let $\mathscr{L}^{q}$ be the matrix obtained from $\mathscr{L}^{g}$ by setting each $l_{i j}$ equal to 1 or 0 depending on whether $l_{i j}$ is associated to $g$ or not. Furthermore, let $L^{g}$ be any link having $\mathscr{L}^{g}$ as its linking matrix. Now it follows from $(2.1)$ that $(-1)^{n-1} \varepsilon(g)=\tilde{\nabla}_{L^{g}}(0)$.

Now suppose that $g$ is not a tree. Then $g$ is either disconnected or misses a vertex of $\Gamma$. For suppose that $g$ is connected but is not a tree. Then $g$ contains some loop. This loop has an equal number of edges and vertices. Adding the remaining edges of $g$ cannot increase the number of vertices beyond the number of edges. Hence $g$ has at most $n-1$ vertices since it has $n-1$ edges. Therefore we may choose a split link $L^{g}$ with linking matrix $\mathscr{L}^{g}$. But the Conway polynomial of a split link is zero and hence $\varepsilon(g)=0$.

Thus it only remains to show that $\varepsilon(g)=1$ if $g$ is a tree. We shall do this by inducting on $n$. If $n=2$ it is shown in [4] that $\tilde{\nabla}_{L}(0)=-l_{12} \cdot{ }^{1}$ This starts the induction. Now suppose that $L$ has $n$ components and that the theorem is true for links with fewer components. Since $g$ is a tree, there is some outermost vertex, say $K_{i}$, which is connected by an outermost edge to $K_{j}$. Now choose $L^{g}$ so that it appears in part as shown in Figure 2.1. Changing and smoothing the indicated crossing as illustrated in the figure gives $\nabla_{L^{g}}(z)=-z \nabla_{L^{\prime}}(z)$ and hence $\tilde{\nabla}_{L^{g}}(0)=$ $-\tilde{\nabla}_{L^{\prime}}(0)$.

\footnotetext{
${ }^{1}$ Note that a slightly different definition of $\nabla_{L}(z)$ is used in that paper than here; namely that, $\nabla_{l}(z)=\operatorname{det}\left(t^{-1} V-t V^{T}\right)$ with $z=t-t^{-1}$.
} 


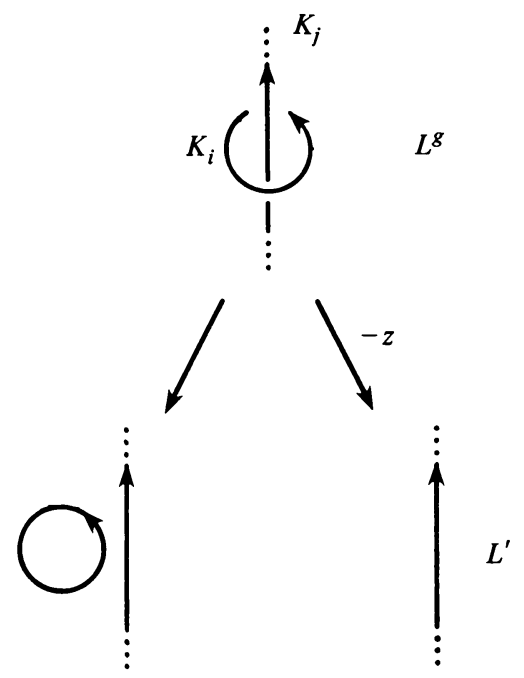

FIGURE 2.1

But $L^{\prime}$ has $n-1$ components and so by our inductive hypothesis, and the fact that $\Gamma\left(L^{\prime}\right)$ has only one subtree $h$ for which $\bar{h} \neq 0$, we have $\tilde{\nabla}_{L^{g}}(0)=(-1)^{n-1}$. Hence $\varepsilon(g)=1$.

As a final remark, note that the number of terms in $\sum_{g \in T} \bar{g}$ is given by $(-1)^{n-1} \overline{\mathscr{L}}_{i j}$, where $\overline{\mathscr{L}}_{i j}$ is the $(i, j)$ cofactor of the matrix $\overline{\mathscr{L}}$ which is obtained from $\mathscr{L}$ by setting the linking numbers equal to 1 . It can easily be shown that this number is $n^{n-2}$.

\section{REFERENCES}

1. J. H. Conway, An enumeration of knots and links, and some of their algebraic properties, Computational Problems in Abstract Algebra, Pergamon Press, New York, 1970, pp. 329-358.

2. C. Giller, A family of links and the Conway calculus, Trans. Amer. Math. Soc. 270 (1982), 75-109.

3. F. Hosokawa, On $\nabla$-polynomials of links, Osaka Math. J. 10 (1958), 273-282.

4. J. Hoste, The Arf invariant of a totally proper link, Topology Appl. 18 (1984), 163-177.

5. L. H. Kauffman, The Conway polynomial, Topology 20 (1981), 101-108.

6. H. Murakami, The Arf invariant and the Conway polynomial of a link, Math. Seminar Notes, Vol. 11, Kobe University, 1983, pp. 335-344.

Department of Mathematics, Rutgers University, New Brunswick, New Jersey 08903 